INPLASY

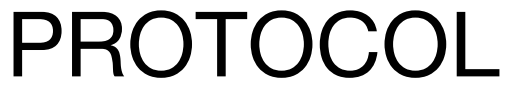

To cite: Busch et al.

Systematic review of OR to ICU handoff standardization interventions highlights need for focus on sustainability and patient outcomes. Inplasy protocol 202220035. doi: 10.37766/inplasy2022.2.0035

Received: 11 February 2022

Published: 11 February 2022

Corresponding author: Julian Conn Busch

julian.connbusch@uchospitals.edu

Author Affiliation:

University of Pennsylvania.

Support: University of

Pennsylvania.

Review Stage at time of this submission: Completed but not published.

Conflicts of interest: None declared.

\section{Systematic review of OR to ICU handoff standardization interventions highlights need for focus on sustainability and patient outcomes}

Busch, JC1; Muralidharan, M2; Wu, J3; Di Taranti, L4; Hernandez, ET5; Collard, M6; Lane-Fall, M7.

Review question / Objective: The objective of this review is to examine if and how interventional studies on handoffs of patients from the operating room (OR) to the intensive care unit (ICU) analyze interventional sustainability and their impacts on patient outcomes.

Eligibility criteria: Inclusion criteria for studies were as follows: (1) publication of the study as a full-text manuscript in a peer-reviewed journal and (2) description of an intervention to standardize the OR to ICU handoff.

Information sources: Information sources are the following electronic databases: ABI Inform, Business Source Complete and HealthBusiness FulIText (EBSCO), CINAHL, ClinicalTrials.Gov, Cochrane Review, EMBASE, Ovid Medline, PubMed, Scopus, and Web of Science.

INPLASY registration number: This protocol was registered with the International Platform of Registered Systematic Review and Meta-Analysis Protocols (INPLASY) on 11 February 2022 and was last updated on 11 February 2022 (registration number INPLASY202220035).

\section{INTRODUCTION}

Review question / Objective: The objective of this review is to examine if and how interventional studies on handoffs of patients from the operating room (OR) to the intensive care unit (ICU) analyze interventional sustainability and their impacts on patient outcomes.

Rationale: We conducted a systematic review to synthesize findings about OR-toICU handoff intervention sustainability and the impact on handoff interventions on patient outcomes, neither of which are well 
described in recent reviews of OR to ICU handoff studies.

Condition being studied: We did not study a medical condition.

\section{METHODS}

Search strategy: Ten databases were used to identify research articles: ABI Inform, Business Source Complete and HealthBusiness FullText (EBSCO), CINAHL, ClinicalTrials.Gov, Cochrane Review, EMBASE, Ovid Medline, PubMed, Scopus, and Web of Science. We searched for synonyms of "operating room," "intensive care unit," and "patient handoff," using both natural language terms and controlled vocabulary when available.

Participant or population: Patients undergoing an operation and subsequently being transferred to an intensive care unit (ICU); clinicians caring for patients who have undergone an operation and who are subsequently transferred to an ICU.

Intervention: Process to guide the handoff that is performed as the patient is transferred from the operating room to the ICU.

\section{Comparator: Standard care.}

Study designs to be included: Quasiexperimental studies, randomized controlled trials.

Eligibility criteria: Inclusion criteria for studies were as follows: (1) publication of the study as a full-text manuscript in a peer-reviewed journal and (2) description of an intervention to standardize the OR to ICU handoff.

Information sources: Information sources are the following electronic databases: $A B I$ Inform, Business Source Complete and HealthBusiness FullText (EBSCO), CINAHL, ClinicalTrials.Gov, Cochrane Review, EMBASE, Ovid Medline, PubMed, Scopus, and Web of Science.
Main outcome(s): Presence and length of sustainability phase, measurement of patient outcomes.

Additional outcome(s): Data collection methods, change in information exchange, clinician satisfaction.

Quality assessment / Risk of bias analysis: We used the Cochrane Consumers and Communication Review Group Study Quality Guide to present and synthesize results using the criteria pertaining to the study format and PRISMA guidelines for reporting.

Strategy of data synthesis: Study data was collected in phases by a single reviewer and verified by a second reviewer. The primary focus of data collection is the presence or absence of a sustainability phase, any measured outcomes in said sustainability phase, the presence or absence of patient outcome data, and any findings reported in the patient outcome data. We did not do a meta-analysis as the significant heterogeneity in the outcomes renders them difficult to collapse into a single measure, therefore data synthesis was not warranted.

Subgroup analysis: We did not perform subgroup analysis.

Sensitivity analysis: We did not perform sensitivity analysis, as the major focus of this review was which outcomes were measured, not the synthesis of the outcome data.

Language: No language limits were imposed.

Country(ies) involved: United States.

Keywords: operating room; postoperative; intensive care unit; critical care; handoff; handover; transfer.

Contributions of each author:

Author 1 - Julian Conn Busch collected data and drafted the manuscript.

Email: julian.connbusch@uchospitals.edu 
Author 2 - Madhavi Muralidharan drafted the manuscript and collected data.

Email:madhavi.muralidharan@pennmedi cine.upenn.edu

Author 3 - Jasmine Wu drafted the manuscript and collected data.

Email: jasmwu@sas.upenn.edu

Author 4 - Laura Di Taranti drafted the manuscript and collected data.

Email: ditaranti-laura@cooperhealth.edu Author 5 - Enrique Torres Hernandez drafted the manuscript and collected data.

Email: torreshernan@uthscsa.edu

Author 6 - Meredith Collard drafted the manuscript and collected data.

Email:meredith.collard@pennmedicine.upe nn.edu

Author 7 - Meghan Lane-Fall is the senior researcher and drafted the manuscript.

Email:meghan.lanefall@pennmedicine.upen n.edu 